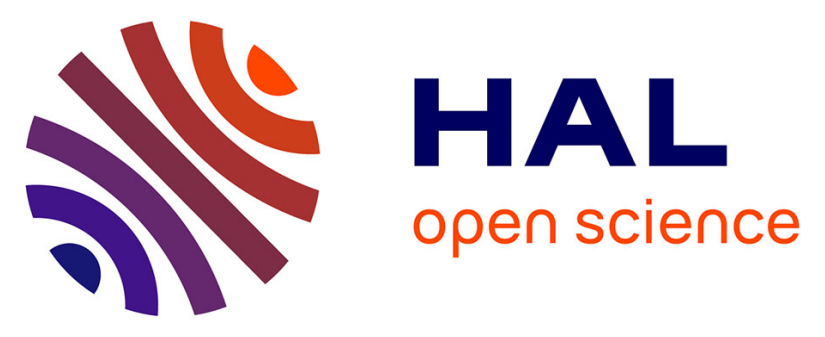

\title{
A Robust Region-based Active Contour for Object Segmentation in Heterogeneous Case
}

\author{
Wassima Ait Fares, El Houssine Bouyakhf, Fakhita Regragui, Ariane
} Herbulot, Michel Devy

\section{- To cite this version:}

Wassima Ait Fares, El Houssine Bouyakhf, Fakhita Regragui, Ariane Herbulot, Michel Devy. A Robust Region-based Active Contour for Object Segmentation in Heterogeneous Case. \&\#1085 / Pattern Recognition and Image Analysis: Advances in Mathematical Theory and Applications, 2014, 24 (1), pp.24-35. 10.1134/S1054661814010027 . hal-02018886

\section{HAL Id: hal-02018886 \\ https://hal.laas.fr/hal-02018886}

Submitted on 5 Mar 2019

HAL is a multi-disciplinary open access archive for the deposit and dissemination of scientific research documents, whether they are published or not. The documents may come from teaching and research institutions in France or abroad, or from public or private research centers.
L'archive ouverte pluridisciplinaire HAL, est destinée au dépôt et à la diffusion de documents scientifiques de niveau recherche, publiés ou non, émanant des établissements d'enseignement et de recherche français ou étrangers, des laboratoires publics ou privés. 


\title{
A Robust Region-Based Active Contour for Object
}

\section{Segmentation in Heterogeneous Case}

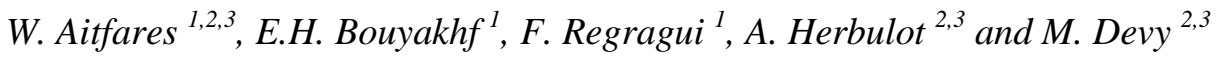 \\ ${ }^{1}$ LIMIARF-FSR, Université Mohammed V-Agdal, B.P 1014 RP, Rabat, Maroc \\ ${ }^{2}$ CNRS ; LAAS ; 7 avenue du Colonel Roche, F-31077 Toulouse Cedex 4, France \\ ${ }^{3}$ Université de Toulouse ; UPS, INSA, INP, ISAE ; UT1, UTM, LAAS ; F-31077, Toulouse, France
}

\begin{abstract}
In this paper, we propose a new approach using a local version of the region-based active contour for object segmentation in images presenting heterogeneity in both the object of interest and the background. The local version was recently developed to deal with heterogeneous appearances by relying on extracting the local instead of global image statistics where the local extracted area is defined by using a disk, sketched at each point along the active contour, with a constant radius size inside and outside the contour. However, the use of a constant radius may prevent the active contour getting more information from its neighborhood and thus being trapped by undesired boundaries. To avoid this error segmentation, the local extracted area using our proposed approach is determined based on using two different radii to extract separately the interior and the exterior local information of the active contour. Using synthetic and real images, our approach shows an improvement in term of computation time and outperforms the conventional methods in noisy images presenting heterogeneity in both the object of interest and the background and using inadequate contour initialization.
\end{abstract}

Keywords: Active contour, Object segmentation, Heterogeneity, Local statistics, Level set.

\section{Introduction}

Active Contour (AC) is a technique that has become very popular and has been widely used in image segmentation $[2-8,12,14-15]$. The main objective of this technique is to segment an object of interest (i.e. Region Of Interest ((ROI)) by iteratively deforming a contour until it reaches the object boundaries by minimizing energy functional. Existing $\mathrm{AC}$ approaches can be generally classified into two categories: edgebased techniques [3, 6] and region-based techniques [2, 4-5, 7, 14-15]. Edge-based AC techniques look for 
intensity discontinuities in the image and use the image gradients to guide the evolution of the contour. They assume that the ROI boundaries can be detected in certain areas that show rapid intensity changes and only consider the information located close to the evolving contour rather than the information located inside and outside it. The main drawbacks of these methods come from their sensitivity to image noise and initial conditions due to the highly localized image information. Recent works in AC have focused on region-based segmentation techniques which aim to identify the desired regions by using a certain region descriptor to guide the motion of the AC.

Region-based techniques, derived from Zhu and Yuille work [15] and based on the user's perceptions on the image content (e.g. intensity, color, or texture), search for uniformity within the region of interest and within the background. These techniques outperform the edge-based methods for their robustness to image noise and their low sensitivity to the initial contour location. However, the region-based methods suffer from limitations due to the assumption on the image intensities which should be statistically homogeneous in the ROI and could also be in the background.

In fact, intensity inhomogeneity often occurs in many real-world images due to different modalities; therefore it will be difficult to maintain the global constraint on the image data. To handle intensity inhomogeneity problems, Lankton and Tannenbaum [7] have considered local instead of global image statistics where the AC is moved based on local information. This local region-based approach showed its capability of segmenting heterogeneous objects that would be difficult to be accurately segmented using a standard global method.

Nonetheless, for standard AC methods, neither the global region-based methods $[4,8,14]$ nor their localizing versions [7] resolve completely the problems encountered in the object segmentation by the AC, mainly in the case of noisy image suffering from heterogeneous characteristics and using inadequate contour initialization. In fact, global region-based methods are robust to contour initialization and image noise, but they are more sensitive to image heterogeneity. In contrast, the local region-based method is robust against the heterogeneity by allowing foreground and background to be described in terms of local regions, but it is more sensitive to contour initialization and image noise and consequently can eventually produce segmentation errors.

In order to overcome the drawbacks of each approach alone (global region-based and local region-based) and cope with the aforementioned limitations, namely, the presence of the heterogeneity (in ROI or in background) in noisy image and the use of an inadequate contour initialization, we developed an approach that combines the local-based and global-based image statistics [2] where the global and local information are both region-based. The combination of local and global information was alternatively addressed by other authors $[11,13]$. Paragios 
and Deriche [11] have proposed a combined energy minimization based on edge-based and region-based energies. Likewise, Sum and Cheung [13] have proposed a combined energy minimization based on the sum of a global region-based and energy based local image contrast. In our approach [2], the hybrid region-based combination depends on where the heterogeneity appears (in ROI or in background). For instance, if the object is heterogeneous compared to the background, the image statistics are extracted locally inside the AC and globally outside it using the technique Local IN-Global OUT presented in [2]; whereas, in the case of heterogeneous background, image statistics are extracted locally outside the $\mathrm{AC}$ and globally inside it using the technique Global IN-Local OUT presented in [2]. The approach presented in [2] was found to be more efficient than the global region-based and the local region-based methods. However, this approach may present limitations in the case of heterogeneity presented in both ROI and background.

To overcome this problem, the object size information and the distance separating the object from its neighbours could be used. In fact, these factors should be considered in the case of heterogeneous characteristics. In Lankton and Tannenbaum contribution [7], a circle with a radius rad is sketched at each point along the AC, and the local selection information is processed by selecting the information interconnected between the disk area (i.e. the region inside the circle), and inside and outside the AC. The choice of the radius size depends on the object scale and the presence of the surrounding clutters. However, using a same radius rad inside and outside the contour prevents to efficiently exploit the object size and the distance separating the object from a possible surrounding nearby neighbors.

In this paper, we propose a local region-based approach that uses two different radii $\left(\mathrm{rad}_{1}\right.$ and $\left.\mathrm{rad}_{2}\right)$ inside and outside the AC, respectively. In fact, the value of $\operatorname{rad}_{i}(i=\{1$ or 2$\})$ strongly depends on the ROI size and the presence of surrounding objects. Thus, in the case of a large object having nearby neighbors, a large radius $\mathrm{rad}_{l}$ is defined inside the AC and a short radius $\mathrm{rad}_{2}$ is defined outside it. While, in the case of a small object with far neighbors, short and large radii are chosen inside and outside the $\mathrm{AC}$, respectively. As a result, the main objectives of this proposed method are (1) to cope with segmenting the object of interest in the case of the presence of heterogeneity in both object and background and (2) to reduce the convergence time of the $\mathrm{AC}$ energy by exploiting the information of the object size as well as the distance separating the object from its nearby neighbors. A variational formulation is presented in which we minimize an energy function to satisfy a certain criterion at each point along the AC.

The rest of the paper is organized as follows. Section 2 presents the terminology used in this paper as well as the AC energy. The selection of the region-based information is presented in Section 3. Section 4 describes the 
proposed approach. Experimental results are presented and discussed in Section 5. Section 6 concludes the paper.

\section{Terminology and active contour energy}

Let $I$ denote a given image defined on the domain $\Omega$ and $I(x)$ the intensity of the pixel $x$ where $x \in \Omega$. One approach to implement the AC segmentation consists in using the Level-Set method which considers the original curve as the zero level of a surface [9-10]. The distortion of the entire surface induces a deformation of the curve shape. This process stimulates the evolution of the AC and achieves, at the end, the object segmentation. Let $C$ be a closed contour represented as the zero level set of a signed distance function $\Phi$, i.e., $C=\{x \mid \Phi(x)=0\}$. The purpose of this process is to implicitly evolve the contour $C$ such that, at the convergence, the distance functions $\Phi<0$ (inside of $C$ ) and $\Phi>0$ (outside of $C$ ) represent the object of interest and the background, respectively. In Level-Set formulation, a smoothed Heaviside function $\mathcal{H} \Phi(x)$ is used to determine the inside and the outside of $C$. The following approximation of the smoothed Heaviside function specifies the interior of $C$ :

$$
\mathcal{H} \Phi(x)=\left\{\begin{array}{lc}
1, & \Phi(x)<-\varepsilon \\
0, & \Phi(x)>\varepsilon \\
\frac{1}{2} \cdot\left(1+\frac{\Phi}{\varepsilon}+\frac{1}{\pi} \cdot \sin \left(\frac{\pi \cdot \Phi(x)}{\varepsilon}\right)\right), & \text { otherwise }
\end{array}\right.
$$

Similarly, the exterior of $C$ is specified as $(1-\mathcal{H} \Phi(x))$.

The energy functional is only computed in a narrow band around $C$ as presented in Adalsteinsson and Sethian work [1] in order to decrease the computational complexity of the standard Level-Set method. To specify this area around the curve, a smoothed version of the Dirac delta (2) which presents the derivative of the Heaviside function is used:

$$
\delta \Phi(x)=\left\{\begin{array}{cc}
1, & \Phi(x)=0 \\
0, & |\Phi(x)|<\varepsilon \\
\frac{1}{2 \cdot \varepsilon} \cdot\left(1+\cos \left(\frac{\pi \cdot \Phi(x)}{\varepsilon}\right)\right), & \text { otherwise }
\end{array}\right.
$$

To perform AC segmentation, we initially define an objective that determines what we want to extract from the image, and then we develop an energy criterion that should be minimized to achieve this objective. The region-based energy is expressed in general by an integral domain of a region descriptor (energy criterion). The choice of a relevant energy expression is a significant aspect that has been widely addressed by several authors as presented in $[4,8,14]$. In this paper, we employ two region-based energies presented in [4] and [14] where the ROI is represented by its mean intensities. The first descriptor used in this paper, introduced by Chan and Vese [4], models the image foreground and background as constant intensities that are represented by their means; it is given by: 


$$
k(x, \Omega)=\left(I(x)-\mu_{\text {in }}\left(\Omega_{\text {in }}\right)\right)^{2}+\left(I(x)-\mu_{\text {out }}\left(\Omega_{\text {out }}\right)\right)^{2} .
$$

where $\Omega_{\text {in }}$ and $\Omega_{\text {out }}$ denote the inside and the outside of the contour $C$, respectively, and $\mu_{\text {in }}$ and $\mu_{\text {out }}$ denote the mean intensities inside and outside $C$, respectively. The second descriptor used in this paper is introduced by Yezzi and al. [14] and expressed as:

$$
k(x, \Omega)=-\frac{1}{2} \cdot\left(\mu_{\text {in }}\left(\Omega_{\text {in }}\right)-\mu_{\text {out }}\left(\Omega_{\text {out }}\right)\right)^{2}
$$

This descriptor assumes that foreground and background regions should have maximal separate mean intensities. Therefore, according to the chosen descriptor, the energy functional in the Level-Set formulation takes the form:

$$
E(\Phi)=\int_{\Omega} \delta \Phi(x) \cdot k(x, \Omega) d x
$$

The evolution equation of $C$ is expressed by:

$$
\frac{\partial \Phi}{\partial t}(x)=\delta \Phi(x) \cdot \nabla k(x, \Omega)+\lambda \cdot \delta \Phi(x) \cdot \operatorname{div}\left(\frac{\nabla \Phi(x)}{|\nabla \Phi(x)|}\right)
$$

where $\nabla$ and div represent the gradient and the divergence operators, respectively. The second term in (6) is used to keep the curve $C$ smooth and it is weighted by a small positive coefficient $\lambda$. Our models are elaborated based upon techniques of curve evolution, statistical function, and Level-Set method.

\section{Selection of the region-based information}

The global region-based approaches present certain robustness against contour initialization and image noise. In fact, they use all information within an image where both image foreground and background are examined simultaneously as illustrated in Fig.1 where the object of interest is shown in black and the area examined is the entire interior and exterior regions of the AC. Generally, the techniques that use global statistics to model regions are very sensitive to heterogeneous characteristics and may produce segmentation errors. To address this issue, Lankton and Tannenbaum [7] have developed a segmentation technique based on information collected from local interior and exterior regions along the AC rather than global regions in order to overcome the assumption that the foreground and background regions should be separated based on their global statistics. Consequently, the only assumption considered is that the interior and exterior regions are locally different where the local area is selected as illustrated in Fig.2. The choice for the local image statistics inside and outside the AC is based on defining a ball function $B$ to mask local regions by overlapping $B$ with inside and outside the AC [7]. This ball function centered at $x$, where $x$ corresponds here to a point on the $\mathrm{AC}$, is expressed as:

$$
B(x, p)= \begin{cases}1, & \|x-p\|<\mathrm{rad} \\ 0, & \text { otherwise }\end{cases}
$$

where $\mathrm{rad}$ is the ball radius and $p$ is a point in $\Omega$ that assigns to $B$ the value 1 on the local region and 0 elsewhere. 
The local selection approach developed in [7] uses one radius inside and outside the AC for each point along this contour. The performance of the object segmentation using this local selection is related to the radius size which is determined by considering the object scale and the proximity of possible surrounding clutters in order to avoid any error segmentation.

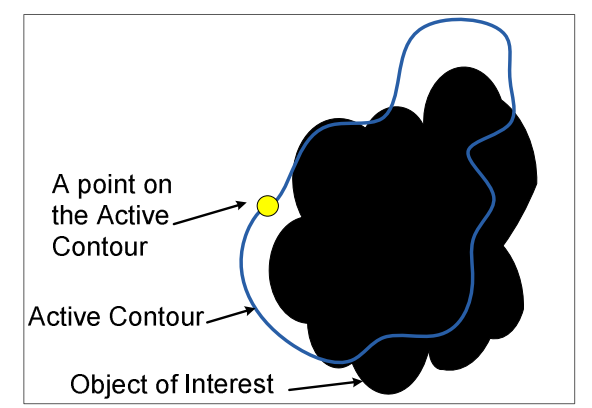

Fig.1 The whole interior and exterior area of the AC is selected for the global region-based approach.

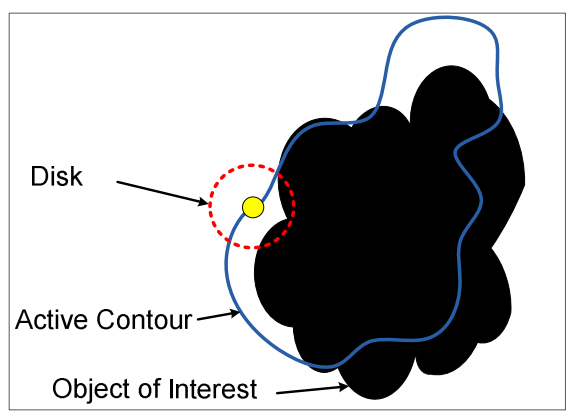

Fig.2 The selected area for the local region-based approach is the intersection of the interior of the disk with the interior and the exterior of the AC.

For information extraction, reducing the selection from global regions to solely local regions leads to place constraints only into selected local regions rather than the whole region of interest and the background. For instance, if such a constraint of homogeneity should be verified, then only those of local regions will be considered to validate this constraint and guide the AC evolution and consequently fewer assumptions are required on the image. We examined here the homogeneity constraint simply as an example of assumptions that the image must verify. For this aim, we employ the energy criteria using (3) and (4) that are seeking to segment homogenous regions by computing the average intensities of the selected regions inside and outside the AC. Other forms of constraints can also be investigated on the image for example verifying a certain variance of intensities within a region or imposing a certain distribution of these intensities, etc.

The approach presented in [7] is still sensitive to the AC initialization and noise. This sensitivity is due to the fact that the information is extracted locally. Thus, with the aim to enable segmenting the object in the case of presence of heterogeneity and using an inadequate AC initialization in noisy image, we proposed an approach in 
[2] that combines both benefits of local-based and global-based techniques using local-based selection in one side of the AC and global-based selection in its other side at each point along the contour. Indeed, depending on where the heterogeneity appears (in ROI or in background), two cases have been investigated in our previous work [2]. Unlike the standard global region-based approaches or the local region-based approach, the approach presented in [2] shows more robustness both against noise and contour initialization thanks to the global statistical information and against heterogeneous appearances thanks to the use of local statistical information. Nevertheless, the approach might present certain limitation in case of a heterogeneous object in a heterogeneous background. To overcome this problem, we develop in this paper an approach derived from the localized regionbased approach using two radii instead of one radius where the size of these radii are determined by considering the ROI size and the distance separating the ROI from its neighbours.

In the following section, we describe the principle of the proposed approach for guiding the AC to the object of interest boundaries in the case of heterogeneity presented in both the object and the background.

\section{Local region-based active contour using two different radii}

The localization radius size may affect the object segmentation result, especially in case of a heterogeneous object in a heterogeneous background. When attempting to capture an object which is of small size with nearby clutter, a small localization radius should be used inside and outside the AC. Similarly, large localization radius inside and outside the AC, is useful when attempting to segment an object which is of large size with far clutters. However, if we desire to segment an Object which is of Large size with Close Neighbors, referred to as OL-CN, or Small size with Far Neighbors, named OS-FN, a small radius is intuitively recommended because either the object has close neighbors or is of a small size. In fact, the use of a small radius can prevent the AC to be trapped by undesired nearby neighbors' boundaries in OL-CN case, or by undesired object interior boundaries coming from the object heterogeneity in case of an OS-FN. In addition, using a same small radius inside and outside the AC will prevent getting additional information for guiding the contour to the actual object boundaries leading thus to the need of more iterations for the AC convergence. To address this situation, we propose an approach that exploits the size of the ROI and the large distance separating the ROI from its possible neighbors using two different radii.

The main distinction between our contribution presented in this paper and those presented in [2] and [7] is that the proposed approach consists on selecting local statistics in both sides (interior and exterior) of the AC by allowing a flexible choice of the radius size where the radius chosen inside the AC is different from the radius 
used outside it. The use of these two different radii is suggested to deal with heterogeneity problems and to decrease the number of iterations for the AC convergence to the ROI boundaries.

Let Large_rad and Short_rad be the two different radii for the two ball functions $B \_L a r g e$ and $B \_S h o r t$, respectively. These two ball functions are centered at $x$, masking local regions, and are expressed as:

$$
\begin{gathered}
B_{-} \operatorname{Large}(x, p)=\left\{\begin{array}{ll}
1, & \|x-p\|<\text { Large_rad } \\
0, & \text { otherwise }
\end{array},\right. \\
B_{-} \operatorname{Short}(x, p)= \begin{cases}1, & \|x-p\|<\text { Short_rad }_{-} \\
0, & \text { otherwise }\end{cases}
\end{gathered}
$$

where $p$ sets $B \_L a r g e$ and $B \_S h o r t$ at 1 on the local region, dependently on which radius is considered (Large_rad or Short_rad, respectively), and 0 elsewhere.

Depending on object size and the distance separating the object from its surrounding neighbors, two cases have to be investigated as discussed below. For instance, in the case of an OS-FN, since the object is of small size and the object neighbors are far, a Short radius Inside the contour and a Large radius Outside the contour, referred to as SILO for short, is recommended. Similarly, in the case of an OL-CN; a Large radius Inside the contour and a Short radius Outside the contour, referred to as LISO for short, is employed. The area considered in each proposed technique (SILO or LISO) is the intersection between the interior area of the local selection, using the two radii, and the interior and the exterior of the $\mathrm{AC}$ for each point along the contour. By doing so, our new approach is able to segment a heterogeneous ROI in a heterogeneous background and outperforms the approach presented in [2] that requires homogeneity in ROI or in background. Moreover, comparison with the approach presented in [7], the use of two radii of different sizes allows the AC to segment the object of interest in a much less reduced computing time; particularly in the case of an OL-CN and an OS-FN.

\subsection{Large size of the object of interest placed with close neighbors}

In OL-CN case, in order to exploit the large size of the object of interest and prevent the AC to be trapped by the boundaries of the object's neighbors, we choose the technique, referred to as, LISO, which employs a large radius inside the $\mathrm{AC}$ and a short radius outside it. Fig.3 illustrates the principle of this technique. The object is designed in black and gray regions to show the heterogeneity in the ROI. The two dotted curves limit the local selection areas using the two different radii inside and outside the AC. The remaining objects present the nearby neighbors of the object of interest to show the heterogeneity of the background. 


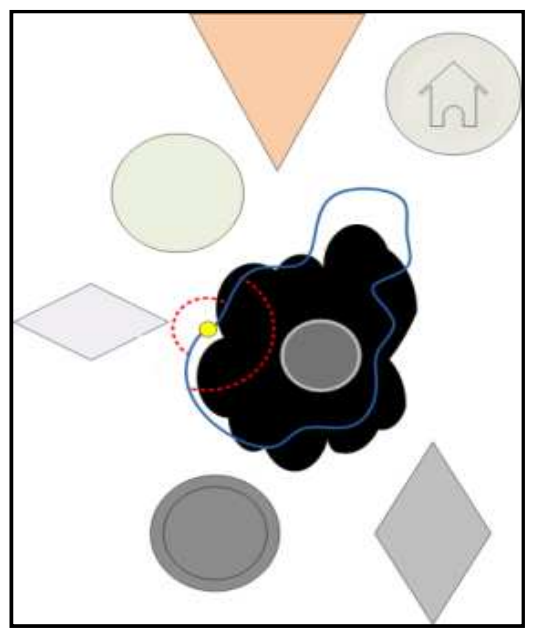

Fig.3 LISO-based technique in the OL-CN case. The object nearly at the center and designed by the gray and black areas represents a large heterogeneous ROI. The surrounding objects represent the neighbors which are close to the ROI. The two red dotted curves limit the local selection using a large radius inside the AC and a short radius outside it.

The energy functional is expressed in this technique as follows:

$$
\begin{gathered}
E(\Phi)=\int_{\Omega_{\text {in }}} \delta \Phi(x) \cdot \int_{\Omega_{p_{\text {LLarge }}}} B \_\operatorname{Large}(x, p) \cdot k\left(p, \Omega_{\text {in }}\right) d p d x+ \\
\int_{\Omega_{\text {out }}} \delta \Phi(x) \cdot \int_{\Omega_{p_{\text {_Short }}}} B \_\operatorname{Short}(x, p) \cdot k\left(p, \Omega_{\text {out }}\right) d p d x,
\end{gathered}
$$

where $\Omega_{p_{-} L a r g e}$ and $\Omega_{p_{-} S h o r t}$ represent the local regions masked by $B \_L a r g e$ and $B \_S h o r t$, respectively, and both ball functions are centered at $x$.

In this case, the evolution of the curve $C$ will be expressed as:

$$
\begin{gathered}
\frac{\partial \Phi}{\partial t}(x)=\delta \Phi(x) \cdot\left[\int_{\Omega_{p_{-} \text {Large }}} B_{-} \operatorname{Larg}(x, p) \cdot \nabla k\left(p, \Omega_{\text {in }}\right) d p+\int_{\Omega_{p_{\text {_Short }}}} B_{-} \operatorname{Short}(x, p) \cdot \nabla k\left(p, \Omega_{\text {out }}\right) d p\right]+ \\
\lambda \cdot \delta \Phi(x) \cdot \operatorname{div}\left(\frac{\nabla \Phi(x)}{|\nabla \Phi(x)|}\right) .
\end{gathered}
$$

\subsection{Small size of the object of interest placed with far neighbors}

In OS-FN case, in order to get benefits from the distance separating the object of interest from its possible neighbors and to obtain more information of the environment surrounding the ROI and thus to speed up the convergence of the $\mathrm{AC}$, we choose the technique, referred to as SILO. As mentioned above, this technique employs a short radius inside the AC and a large radius outside it. Fig.4 illustrates the principle of this technique. 


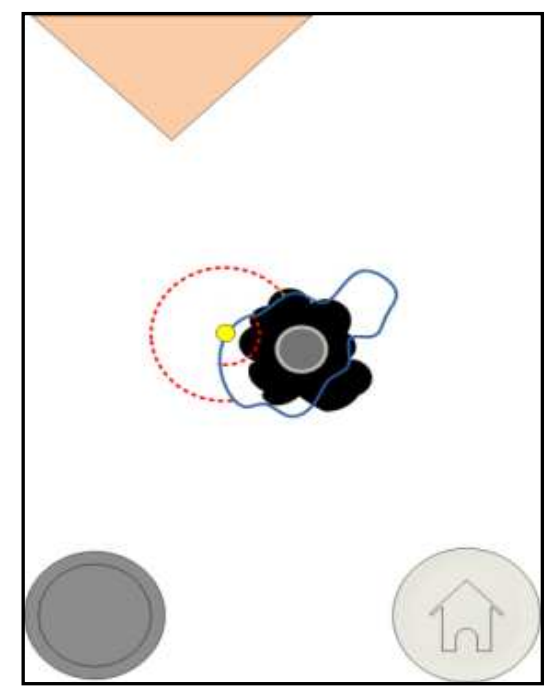

Fig.4 SILO-based technique in the OS-FN case. The object nearly at the center and designed by the gray and black areas represents a small heterogeneous ROI. The surrounding objects represent the neighbors which are far from the ROI. The two red dotted curves limit the local selection using a short radius inside the AC and a large radius outside it.

In this case, the energy functional is expressed as follows:

$$
\begin{gathered}
E(\Phi)=\int_{\Omega_{\text {in }}} \delta \Phi(x) \cdot \int_{\Omega_{p_{\text {_ Short }}}} B \_\operatorname{Short}(x, p) \cdot k\left(p, \Omega_{\text {in }}\right) d p d x+ \\
\int_{\Omega_{\text {out }}} \delta \Phi(x) \cdot \int_{\Omega_{p_{\text {LLarge }}}} B \_\operatorname{Large}(x, p) \cdot k\left(p, \Omega_{\text {out }}\right) d p d x
\end{gathered}
$$

and the evolution of the curve $C$ is expressed as:

$$
\begin{gathered}
\frac{\partial \Phi}{\partial t}(x)=\delta \Phi(x) \cdot\left[\int_{\Omega_{p_{-} \text {Short }}} B_{-} \operatorname{Short}(x, p) \cdot \nabla k\left(p, \Omega_{\text {in }}\right) d p+\int_{\Omega_{p_{-L \text { Large }}}} B_{-} \operatorname{Larg}(x, p) . \nabla k\left(p, \Omega_{\text {out }}\right) d p\right]+ \\
\lambda \cdot \delta \Phi(x) \cdot \operatorname{div}\left(\frac{\nabla \Phi(x)}{|\nabla \Phi(x)|}\right) .
\end{gathered}
$$

Using these above mentioned techniques LISO and SILO in cases of OL-CN and OS-FN, respectively, allows the object segmentation approach to: (1) assure robustness against heterogeneity that can appear in ROI or in background and robustness against AC initialization and noise as the work in [2]. (2) give more flexibility to the approach so that it can be applied even in case of presence of heterogeneity in both the ROI and the background which presents a performance improvement compared to the method developed in [2]. (3) Reduce the computation time for the AC convergence by selecting a flexible size of the radius inside and outside the AC and getting more information on the ROI size and its surrounding environment; which presents a performance improvement compared to the approach presented in [7]. 


\section{Experimental results}

In order to assess the performance of the proposed approach; experiments have been conducted on different sets of synthetic and real image data. In these experiments, the proposed method is compared with the conventional methods in terms of segmentation accuracy in noisy images using different types of noise and using an inadequate $\mathrm{AC}$ initialization. The proposed approach is evaluated in images presenting heterogeneity in both the ROI and the image background.

In the following two subsections, we evaluate the performance of the proposed approach and examine the advantage of using two different radii in the case of presence of heterogeneity in both the ROI and the background for synthetic and real noisy images. It is worth mentioning that the choice of the SILO-based technique or the LISO-based technique is made according to the OS-FN or OL-CN case, respectively. A comparative performance evaluation for this proposed approach with other conventional segmentation methods using the descriptors presented in [4] and [14] is presented and discussed below.

\subsection{Object segmentation in synthetic images}

Fig.5 presents the results related to the OS-FN case in a synthetic image. Fig.5 (a) represents the original synthetic image. Fig.5 (b-h) represent the same image in which we added a Poisson distribution noise. The object is an OS-FN with heterogeneous characteristics which is presented by the gray and white intensities and is located nearly in the image center. The surrounding objects present the ROI neighbors to show the heterogeneity of the background. By using an inadequate initialization of the contour as shown in Fig.5 (b), Fig.5 (c-h) illustrate the ROI segmentation results using different approaches based on the Chan and Vese energy criterion [4]. Fig.5 (c) presents the segmentation result using the global approach that fails due to the presence of heterogeneous characteristics in ROI and background. Using the local approach, the AC was trapped by the internal false ROI boundaries instead of resting on the real exterior ROI boundaries as shown in Fig.5 (d). In addition, due to the presence of heterogeneity in both the ROI and the background, respectively, the Global INLocal OUT and Local IN-Global OUT techniques, presented in [2], were not able to guide the AC to the actual ROI boundaries as shown in Fig.5 (e-f). The segmentation also fails in the case of LISO-based technique (Fig.5 (g)) due to the use of a large radius inside the AC and a short radius outside it. In fact, the evolution of the AC was trapped by the false interior ROI boundaries. In contrast, the SILO-based technique gives accurate object segmentation and maintains the robustness against heterogeneous characteristics, appearing in both ROI and background, by increasing the radius size outside the AC to benefit from the large distance between the ROI and 
its neighbors (Fig.5 (h)). By doing so, more information was exploited from the ROI neighborhood and thus the $\mathrm{AC}$ is protected from being attracted by non-desired contours presented in the interior of the object of interest.

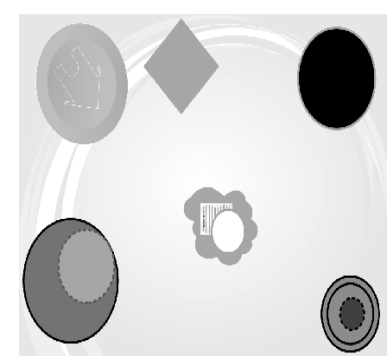

(a) Original image

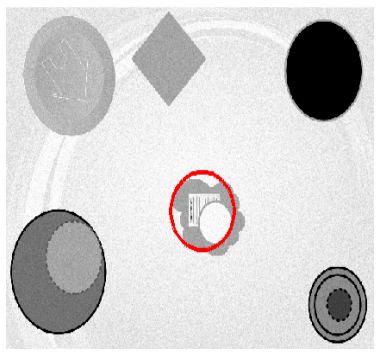

(b) Initialization

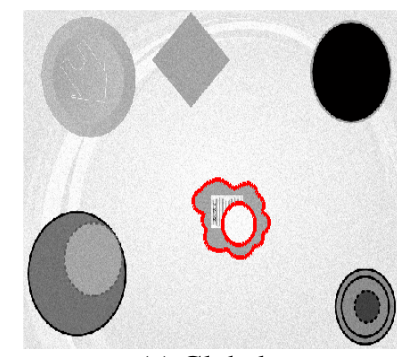

(c) Global

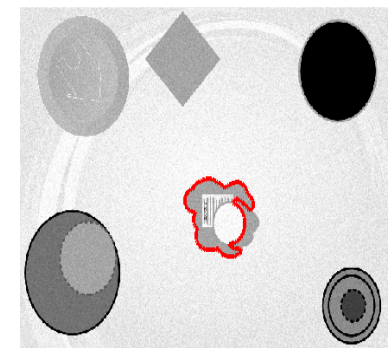

(d) Local

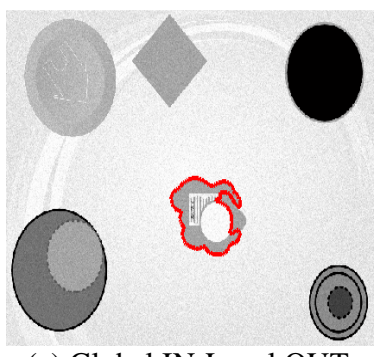

(e) Global IN-Local OUT

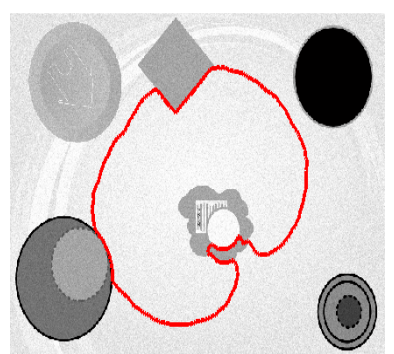

(f) Local IN-Global OUT

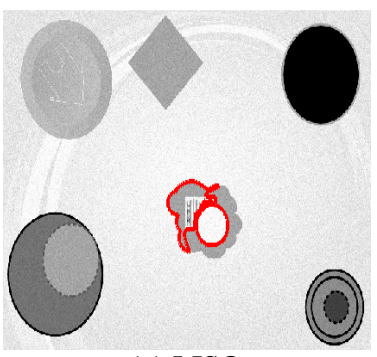

(g) LISO

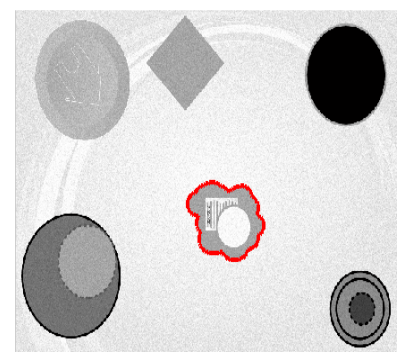

(h) SILO

Fig.5 Segmentation of a heterogeneous object in a heterogeneous background using different approaches for the OS-FN case in a synthetic image with an added "Poisson" distribution noise in (b-h). The original image is represented in (a). The ROI, which is of small size, is represented by the gray and white areas and is located nearly in the image center. The surrounding objects represent the neighbors which are far from the ROI.

Fig.6 illustrates the results obtained in the case of an OL-CN in a synthetic image. Fig.6 (a) represents the original synthetic image while Fig.6 (b-h) represent the same image in which we added a Poisson distribution noise. The ROI presents heterogeneous characteristics as described in gray and white intensities and is located close to the image center. The surrounding objects present the ROI neighbors to show the heterogeneity of the background. By using an inadequate AC initialization as shown in Fig.6 (b), Fig.6 (c-h) illustrate the object segmentation results using different approaches based on the Chan and Vese energy criterion [4]. The global, Global IN-Local OUT and Local IN-Global OUT approaches fail to segment the object of interest due to the heterogeneity in both ROI and background, as shown in Fig.6 (c) and Fig.6 (e-f), respectively. Using the local approach, the contour was attracted this time by false contours corresponding to ROI neighbor's boundaries as shown in Fig.6 (d). Fig.6 (g) and Fig.6 (h) display the segmentation performance using the SILO-based and LISO-based techniques, respectively. Using the SILO-based technique, the AC in this OL-CN case was not able to detect the real ROI boundaries. That was obviously anticipated since this technique uses a short radius inside the AC and a large radius outside it which leads the AC to be attracted by the ROI neighbor's boundaries. The 
LISO-based technique gives however an accurate object segmentation performance and maintains its robustness against heterogeneous attributes, appearing in both the object of interest and the background, by increasing the radius size inside the AC. This allows taking advantage from the large size of the ROI and thus protecting the AC from the close boundaries corresponding to ROI surrounding neighbors. Therefore, our proposed LISObased technique drives the AC to the ROI boundaries and prevents it to be attracted by the ROI neighbor's boundaries since more information from the $\mathrm{AC}$ interior area is used allowing thus the $\mathrm{AC}$ to converge to the actual desired object of interest boundaries.

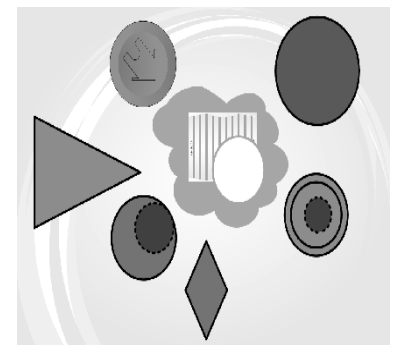

(a) Original image

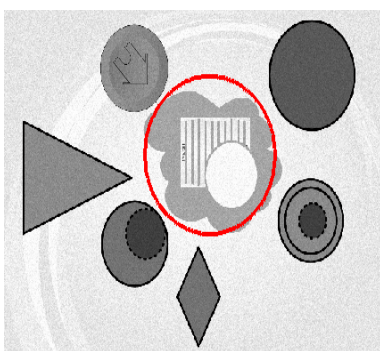

(b) Initialization

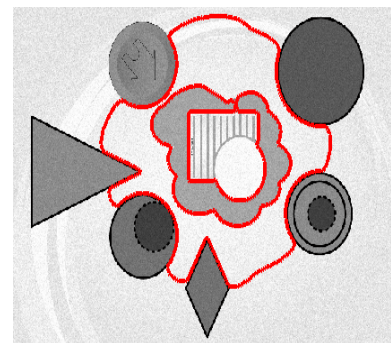

(c) Global

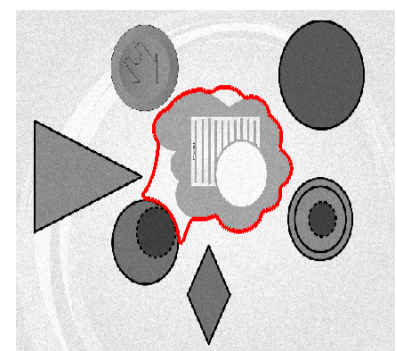

(d) Local

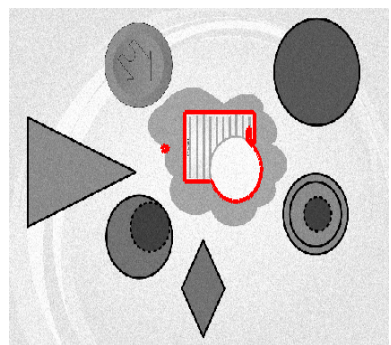

(e) Global IN-Local OUT

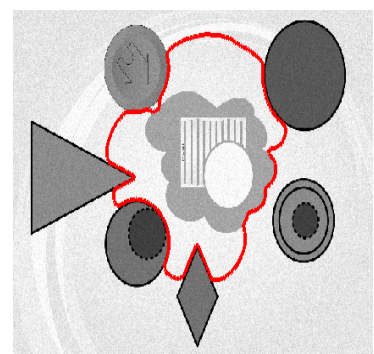

(f) Local IN-Global OUT

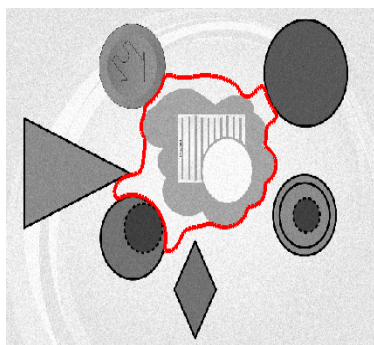

(g) SILO

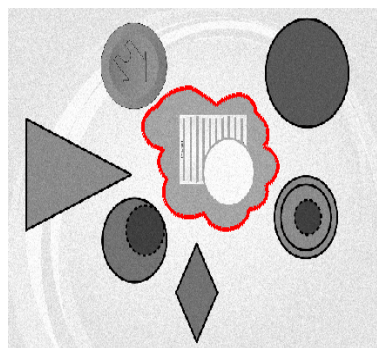

(h) LISO

Fig.6 Segmentation of a heterogeneous object in a heterogeneous background using different approaches for the OL-CN case in a synthetic image with an added "Poisson" distribution noise in (b-h). The original image is represented in (a). The ROI, which is of large size, is represented by the gray and white areas and is located nearly in the image center. The surrounding objects represent the neighbors which are close to the ROI.

\subsection{Object segmentation in real images}

In the case of real images, segmentation results are presented in Fig.7 and Fig.8. In the case of OL-CN, Fig.7 (a) shows original real image and Fig.7 (b-h) show same image corrupted with a "Salt \& Pepper" noise distribution, in which the car represents the object of interest. Notice that even if there is an absence of nearby objects for the ROI in this real image, some zones in the background verifying the energy criterion may play the same role as the presence of ROI close neighbors and they can attract the AC to their boundaries. By using an inadequate AC initialization (Fig.7 (b)), Fig.7 (c-h) illustrate the object segmentation results using different approaches based on the criterion introduced in Chan and Vese work [4]. Using the global approach, the 
heterogeneous characteristics always prevent the $\mathrm{AC}$ to evolve to the ROI boundaries and consequently lead to error segmentation as shown in Fig.7 (c). The object segmentation using the local approach is degraded as shown in Fig.7 (d) due to the sensitivity of this approach to initial contour and also to the presence of certain zones in background that verify the energy criterion. Consequently, some parts of the initial AC sketched far from the real object boundaries easily diverge from the ROI. In both approaches Global IN-Local OUT and Local IN-Global OUT (presented in [2]), the interior and exterior global selection influence the calculated energy value and that leads to an incorrect object segmentations as shown in Fig.7 (e) and Fig.7 (f), respectively, due to the heterogeneous characteristics in both ROI and background. Fig.7 (g) and Fig.7 (h) present the segmentation performance using $S I L O$-based and $L I S O$-based techniques, respectively. The $S I L O$-based technique prevents the AC to be attracted by the real object boundaries since this technique is intended to be used for an OS-FN case and not for an OL-CN case. The segmentation error is due to using a large radius outside the AC, which lead this contour to be attracted by some zones, belonged to the image background, that verify the energy criterion. The LISO-based technique gives however an accurate object segmentation result and maintains its robustness against heterogeneous attributes, appearing in both ROI and background. This was made possible by increasing in this case the radius size inside the $\mathrm{AC}$ since the $\mathrm{ROI}$ is of large size and thus protecting the $\mathrm{AC}$ from the close boundaries corresponding, for this example, to zones belonging to the background.

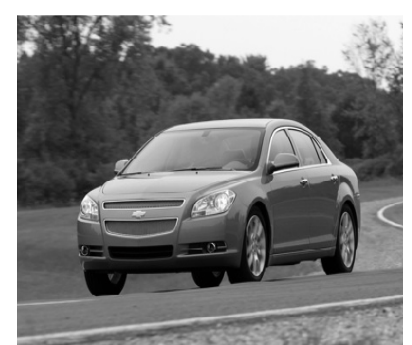

(a) Original image

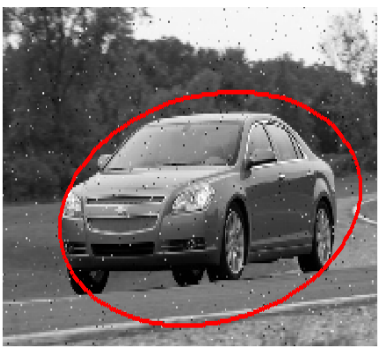

(b) Initialization

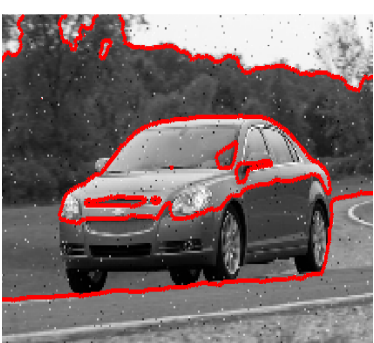

(c) Global

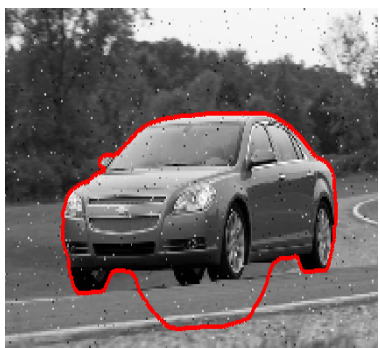

(d) Local

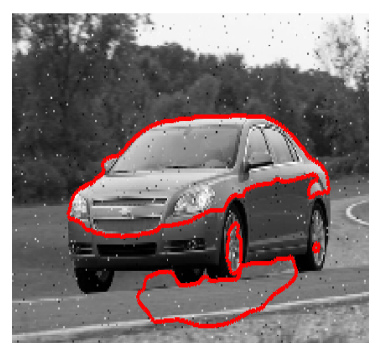

(e) Global IN-Local OUT

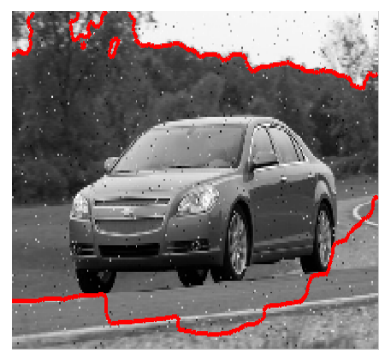

(f) Local IN-Global OUT

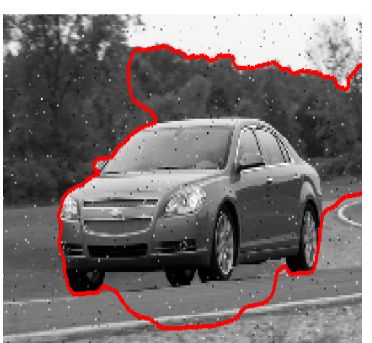

(g) SILO

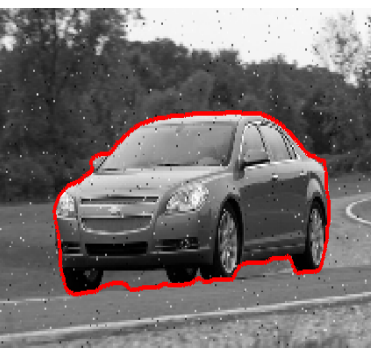

(h) LISO

Fig.7 Segmentation of a heterogeneous object in a heterogeneous background using different approaches for the OL-CN case in a real image with an added "Salt \& Pepper" distribution noise in (b-h). The original image is represented in (a). The car represents the ROI. 
To study the object segmentation in OS-FN real image case, Fig.8 (a) shows original real image and Fig.8 (bh) show same image corrupted with a Gaussian noise distribution. The object of interest is represented by the light gray car. By using an inadequate AC initialization (Fig.8 (b)), Fig.8 (c-h) illustrate the object segmentation results using different approaches based on the descriptor introduced by Yezzi and al. [14]. As shown in Fig.8 (c), the global approach fails to segment the object due to the heterogeneous characteristics in both the ROI and the background. Likewise, in the local approach (Fig.8 (d)), being sensitive to the AC initialization and noise, the selection which is made only locally, prevents the AC to rest on the whole ROI boundaries. Using the techniques Global IN-Local OUT and Local IN-Global OUT, the AC was trapped by the heterogeneity presented in both the ROI and the background, as shown in Fig.8 (e) and Fig.8 (f), respectively. Fig.8 (g) and Fig.8 (h) present the segmentation performance using LISO-based and SILO-based techniques, respectively. The LISO-based technique fails to correctly segment the object of interest. This was expected since this technique, intended to be used for an OL-CN case and not for an OS-FN case, employs a large radius inside the AC which leads this latter to be trapped by the interior ROI heterogeneity. In contrast, the SILO-based technique gives accurate object segmentation and maintains the robustness against heterogeneous attributes, appearing in both the ROI and the background. The outperformance of the segmentation, using our SILO-based technique, resides in receiving more information from the ROI surrounding environment and that by increasing the radius size by some pixels outside the AC.

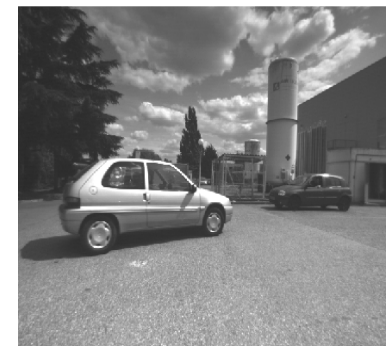

(a) Original image

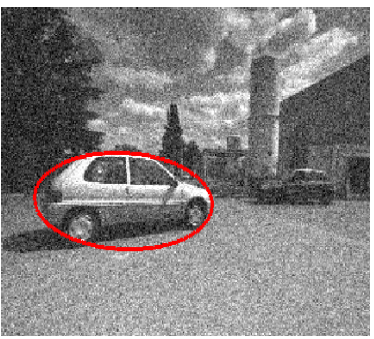

(b) Initialization

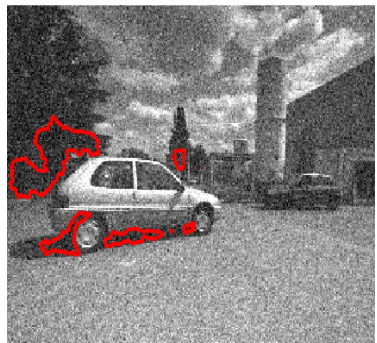

(c) Global

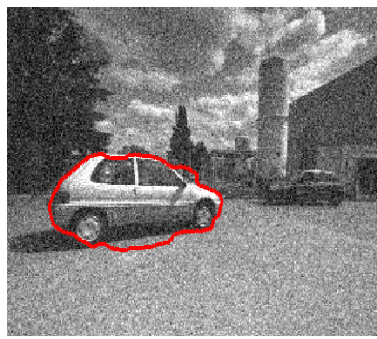

(d) Local

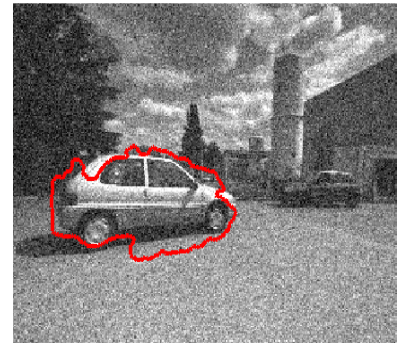

(e) Global IN-Local OUT

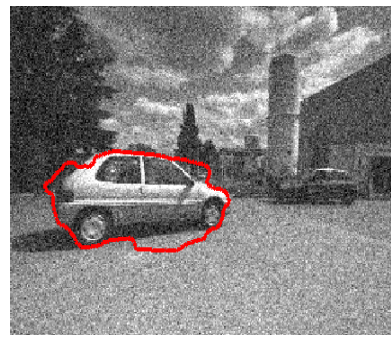

(f) Local IN-Global OUT

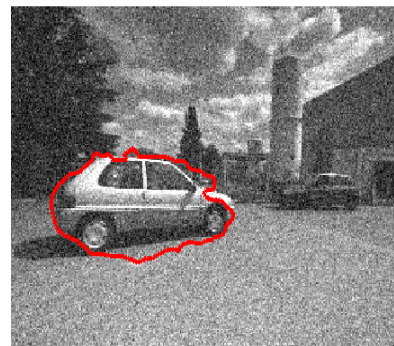

(g) LISO

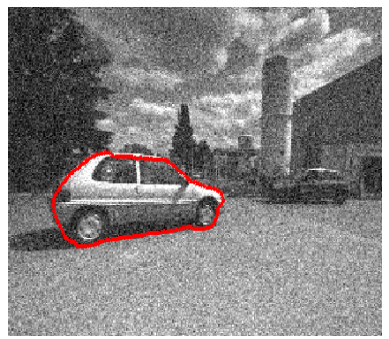

(h) SILO

Fig.8 Segmentation of a heterogeneous object in a heterogeneous background using different approaches for the OS-FN case in a real image with an added "Gaussian" distribution noise in (b-h). The original image is represented in (a). The light gray car represents the ROI. 
To study the performance of the proposed approach in terms of the number of iterations needed to the convergence of the AC energy, a comparative study for the local approach and the proposed approach using an inadequate and adequate contour initialization is discussed in the following subsection.

\subsection{Computational time and energy convergence}

Based on the previous segmentation results obtained with the local and SILO-based approaches as shown in Fig.8 (d) and Fig.8 (h), we studied their performances in terms of the energy convergence by considering the cases of using inadequate and adequate contour initialization as well. Fig.9 shows that for inadequate contour initialization, the SILO-based approach outperforms the local approach; since the minimum of the energy achieved had a smaller value, leading thus to more accuracy in segmentation (as found in Fig.8 (h) compared to Fig.8 (d)) even though it convergences much slower.

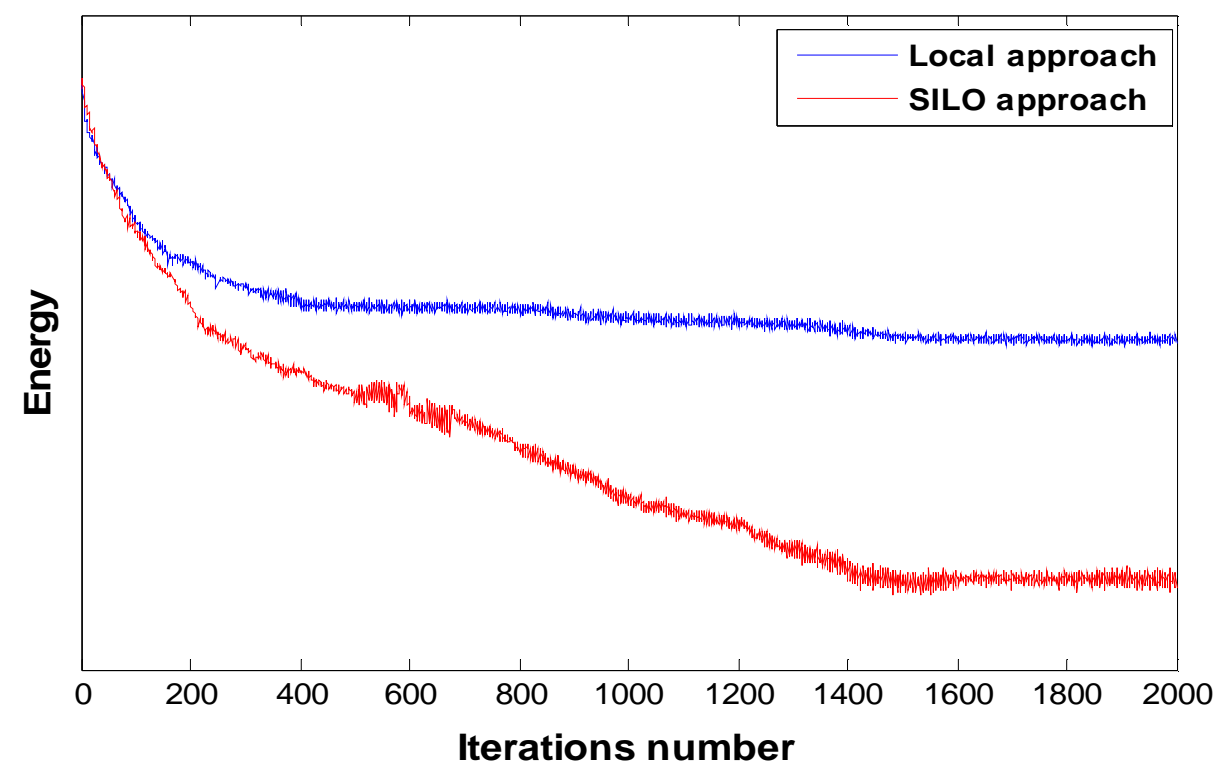

Fig.9 Energy convergence versus the number of iterations for the car segmentation presented in Fig. 8 for the local (blue curve) and SILO (red curve) approaches based on using an inadequate AC initialization.

Fig.10 presents results for adequate contour initialization which reveal that the local approach shows some segmentation improvement compared to the case of using inadequate contour initialization, however it remains less accurate than the segmentation by the SILO-based technique. This is also proven by the energy curves represented in Fig.11 showing that the SILO-based technique converges to a lower value of the energy. In addition, the proposed technique is faster than the local method; it requires less than 400 iterations compared to more than 500 iterations needed using the local approach. In fact, the number of iterations is reduced by our proposed approach by exploiting the information from the ROI neighborhood by increasing the radius size outside the AC. 


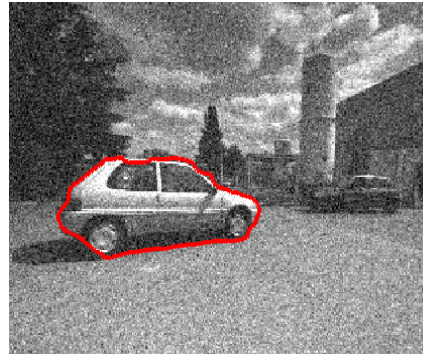

(a) Local

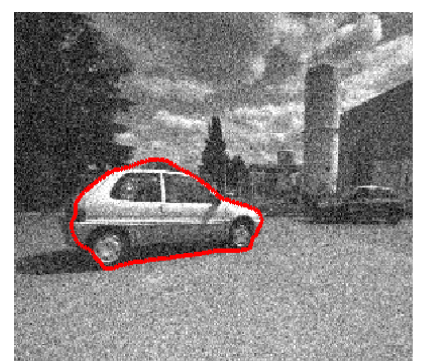

(b) SILO

Fig.10 Car segmentation using the local and SILO approaches based on an adequate AC initialization.

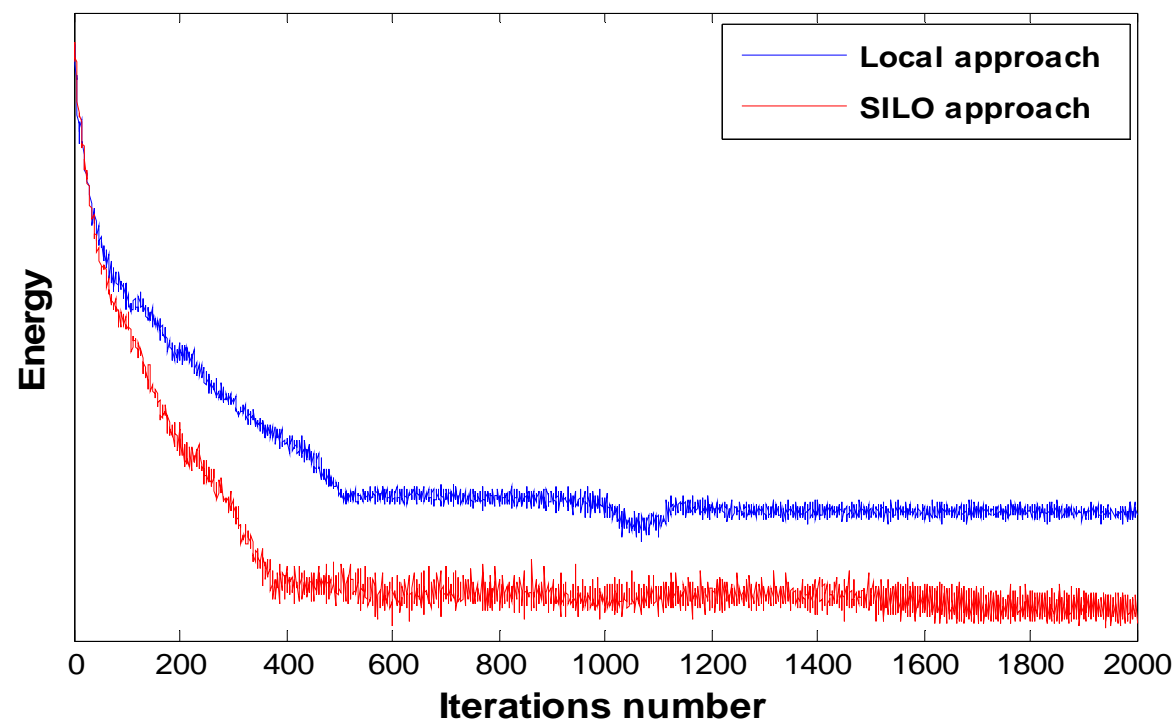

Fig.11 Energy convergence versus the number of iterations for the car segmentation presented in Fig.10 for the local (blue curve) and SILO (red curve) approaches based on using an adequate AC initialization.

\section{Conclusion}

In this paper, a new approach using region-based $\mathrm{ACs}$ for object segmentation in images presenting heterogeneity in both the object of interest and the background has been proposed. This approach derives from the local region-based approach and relies on two different radii instead of only one radius. By using two different radius sizes inside and outside the AC, information on the ROI size and the distance separating the ROI from its neighbors can be advantageously exploited. We used two different criteria for the AC energy to show that the proposed approach can be applied with different energy expressions. We have tested the method empirically on synthetic and real image database and obtained promising results in case of noisy images presenting heterogeneity in both the ROI and the background using inadequate and adequate contour initialization. 


\section{References}

[1] D. Adalsteinsson and J.A. Sethian, A Fast Level Set Method for Propagating Interfaces, J. Comp. Phys. , 1995, pp. 269-277.

[2] W. Aitfares, A. Herbulot, M. Devy, E.H. Bouyakhf and F. Regragui, A novel region-based active contour approach relying on local and global information, in Proc. of IEEE International Conference on Image Processing (ICIP), Bruxelles, Belgique, 2011, pp. 1049-1052.

[3] V. Caselles, R. Kimmel and G. Sapiro, Geodesic actives contours, Int. J. Comput. Vision, vol. 22, no. 1, 1997, pp. 6179.

[4] T. Chan and L. Vese, Active contours without edges, IEEE Trans. Image Process., vol. 10, no. 2, 2001, pp. $266-277$.

[5] D. Cremers, T. Rousson and R. Deriche, A review statistical approaches to level set segmentation: Integrating color, texture, motion and shape, Int. J. Comput. Vision, vol. 72, no. 2, 2007, pp. 195-215.

[6] M. Kass, A. Witkin, and D. Terzopoulos, Snakes: Active contour models, Int. J. Comput. Vision, vol. 1, no. 4, 1988, pp. 321-332.

[7] S. Lankton and A. Tannenbaum, Localizing region-based active contours, IEEE Trans. Image Process., vol. 17, no. 11, 2008, pp. 2029-2039.

[8] O. Michailovich, Y. Rathi and A. Tannenbaum, Image segmentation using active contours driven by the Bhattacharyya gradient flow, IEEE Trans. Image Process., vol. 15, no. 11, 2007, pp. 2787-2801.

[9] S. Osher and R. Fedkiw, Level Set Methods and Dynamic Implicit Surfaces, New York: Cambridge Univ. Press, 2003.

[10] S. Osher and R. Tsai, Level Set Methods and their applications in image science, Commun. Math. Sci., vol. 1, no. 4, 2003, pp. 1-20.

[11] N. Paragios and R. Deriche, Geodesic active regions: A new framework to deal with frame partition problems in computer vision, Int. J. Comput. Vision, vol. 46, no. 3, 2002, pp. 223-247.

[12] Y. Rathi, N. Vaswani, A. Tannenbaum and A. Yezzi, Tracking Deforming Objects using Particle Filtering for Geometric Active Contours, IEEE Trans. Pattern Anal. Match. Intell, vol. 29, no. 8, 2007, pp. 1470-1475.

[13] K. Sum and P. Cheung, Vessel extraction under non-uniform illumination: A level set approach, IEEE Trans. Biomed. Eng., vol. 55, no. 1, 2008, pp. 358-360.

[14] J.A. Yezzi, A. Tsai, and A. Willsky, A fully global approach to image segmentation via coupled curve evolution equations, J. Vis. Comm. Image Rep., vol. 13, no. 1, 2002, pp. 195-216.

[15] SC. Zhu and A. Yuille, Region competition: Unifying snakes, region growing, and bayes/mdl for multiband image segmentation, IEEE Trans. Pattern Anal. Match. Intell, vol. 18, no. 9, 1996, pp. 884-900. 

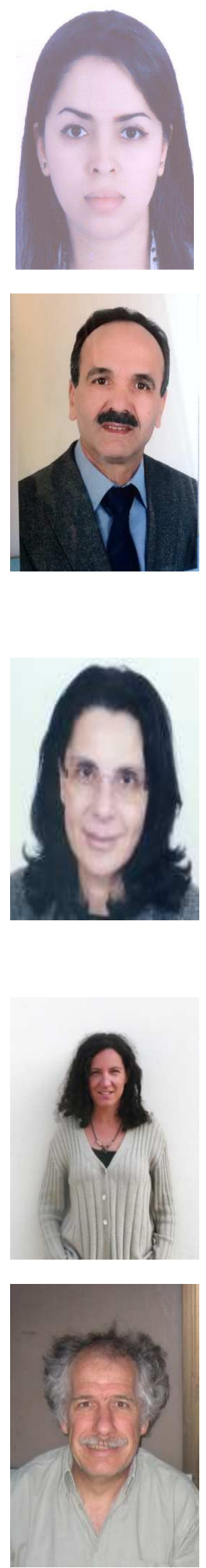

Wassima Aitfares received the B.S and the M.Sc degrees from Mohammed V University, Rabat, Morocco, respectively, in Mathematics in 2005, and Informatics, Telecommunications and Multimedia in 2007. She received her Ph.D. degree from Mohammed V University, Morocco with collaboration with Paul Sabatier University, Toulouse, France in 2013. Her research interests include Image segmentation, Object detection and tracking.

El Houssine Bouyakhf graduated from Sup'Aéro Ecole Nationale Supérieure de l'Aéronautique et de l'Espace (National Higher School of Aeronautics and Space), Toulouse, France, and received the Ph.D degree in Robotics and Artificial Intelligence from LAAS of CNRS and University Paul Sabatier, Toulouse, France. He works as Full Professor at the Faculty of Sciences, Rabat, teaching Computer Sciences, Pattern Recognition, Image Processing and Artificial Intelligence courses respectively at undergraduate and graduate levels. His main topics of interest are: Artificial Intelligence, Computer Vision and Image Processing and Telecommunications. His research focuses on all aspects of Constraint Programming in the Artificial Intelligence field and in the Vision and Robotic field, on fixed and video image processing, object tracking and robotics applications. He is the scientific leader of LIMIARF Lab. (Laboratory of Computer Sciences Artificial Intelligence and Pattern Recognition). He has been the Director or Co-Director of more than $30 \mathrm{PhD}$ students. He is the leader or the key person of several national and international projects and he co-authored over 200 scientific papers (journal articles, book chapters and conference publications).

Fakhita Regragui received the Master degree in Electronics from the Faculty of Sciences at Mohammed V University -Agdal, Rabat, Morocco in 1977 and the $\mathrm{PhD}$ degree in Signal processing and Communications from Rutgers University, New Brunswick, New Jersey, USA in 1990. Worked, from 1977 to 1983, as Assistant professor at the Faculty of Sciences teaching courses related to the Electronics field. Since 1990, she worked as Full Professor at the same Faculty teaching Electronics and Digital Signal Processing courses respectively at undergraduate and graduate levels. Her research interests include applications of signal and image processing in the biomedical field. In signal processing, she is currently working on filtering and classification using different techniques (adaptive filtering, wavelet analysis, neural networks,..) and their applications to neuroscience. In the field of image processing, she is currently working on different tools such as contour detection and texture analysis for different image processing applications (mammograms interpretation, estimation of fish growth parameters, motion tracking applied to robotics).

Ariane Herbulot is an Associate Professor from University Paul Sabatier (Toulouse, France). She obtained her PhD in 2007 at University of Nice-Sophia Antipolis (France) on image segmentation by active contours. She is interested on detection and object tracking for video-surveillance and robotics applications. Her research concerns segmentation, detection, object recognition and motion estimation and tracking.

Michel Devy is Research Director at /Laboratoire d'Analyse et d'Architecture des Systèmes/ of CNRS (LAAS-CNRS) in Toulouse (France): he is team leader of a research group, entitled /Robotics, Action and Perception/ (about 30 persons). For more than 30 years, he has participated to the LAAS Robotics department; his research has been devoted to the application of computer vision in Automation and Robotics. He was involved in numerous national and international projects, about Manufacturing, Mobile Robots for space exploration and for civil safety, 3D Modeling, Intelligent Vehicles, Service robotics ....His current research interests concern at first Perception for Mobile Robots in natural or indoor environments, e.g. visual-based navigation and manipulation, 3D object modelling, Simultaneous Localization and Mapping (SLAM), Design and Implementation of integrated sensors .... and secondly, Applications of robotics technologies on Non Destructive Test, Intelligent Transportation Systems...He was PhD advisor or co-advisor for about $40 \mathrm{PhD}$ students and co author of about 200 scientific communications. 